\title{
Self propulsion due to oscillations on the surface of a cylinder at low Reynolds number
}

\author{
J.R. Blake
}

\begin{abstract}
The two-dimensional flow around an infinite cylinder at low Reynolds number has interested fluid dynamicists for many years. In this paper it is shown that an infinite cylinder can propel itself through a viscous fluid (for example micro-organisms) if it has certain undulations on its surface.
\end{abstract}

\section{Introduction}

It is well known, that for two-dimensional flow around an infinite cylinder at very low Reynolds number, there is no solution which satisfies the no-slip condition on the surface of the cylinder and tends to a finite stream at infinity (Stokes paradox). In this paper a similar problem that may have some applications in the theory behind propulsion of micro-organisms is treated. The solution to the two-dimensional problem of flow due to a cylinder with oscillations on its surface at very low Reynolds number is discussed. These oscillations are caused by a wave passing around the cylinder, and for generality we include both radial and angular oscillations in these movements. The cylinder is propelled at a constant steady velocity through the fluid. For convenience we take axes fixed in the moving organism.

The rate of change of momentum is zero, which indicates that the body

Received 4 May 1971. The author acknowledges the support of a George Murray Scholarship from the University of Adelaide and a studentship from CSIRO of Australia for this research. Comments and suggestions from Professor Sir James Lighthill are appreciated. 
exerts no net force on the fluid and hence allows us to equate the drag term to zero (this is the two-dimensional stokeslet which is the $\log x$ term in the series expansion for the velocity). This then enables us to obtain the velocity of propulsion.

\section{Equations of motion}

The equations of motion for this problem are those for creeping flow,

(1)

$$
\nabla p=\mu \nabla^{2} \mathrm{u}
$$

$$
\nabla \cdot u=0,
$$

where $\mu$ is the viscosity, $p$ the pressure and $u$ the velocity vector. In this two-dimensional problem, it is more convenient to define these equations in terms of the stream function $\psi$ by,

$$
\nabla^{4} \psi=0
$$

where $\nabla^{2}$ is the Laplacian, defined in cylindrical polar coordinates as,

$$
\nabla^{2}=\frac{\partial^{2}}{\partial r^{2}}+\frac{1}{r} \frac{\partial}{\partial r}+\frac{1}{r^{2}} \frac{\partial^{2}}{\partial \theta^{2}} \text {. }
$$

The velocity components in terms of $\psi$ are,

$$
u=\frac{1}{r} \frac{\partial \psi}{\partial \theta}, \quad v=-\frac{\partial \psi}{\partial r},
$$

where $u$ and $v$ are the radial and angular velocities respectively.

Boundary conditions on the cylinder $r=a$ (see Figure 1, p. 257) are given in terms of the surface coefficients $A_{n}$ and $B_{n}$. Thus,

$$
u(a, \theta)=\sum_{n=0}^{\infty} A_{n} \cos n \theta, v(a, \theta)=\sum_{n=1}^{\infty} B_{n} \sin n \theta .
$$

Equation (4) gives rise to the effective slip velocity around the cylinder. The coefficients $A_{n}$ and $B_{n}$ in (4) are obtained later in terms of the deformations to the surface of the cylinder.

We require $v$ to be an odd function in $\theta$

$$
v(r, \theta)=-v(r,-\theta),
$$

so that streaming past the cylinder can occur. It was stated earlier in 


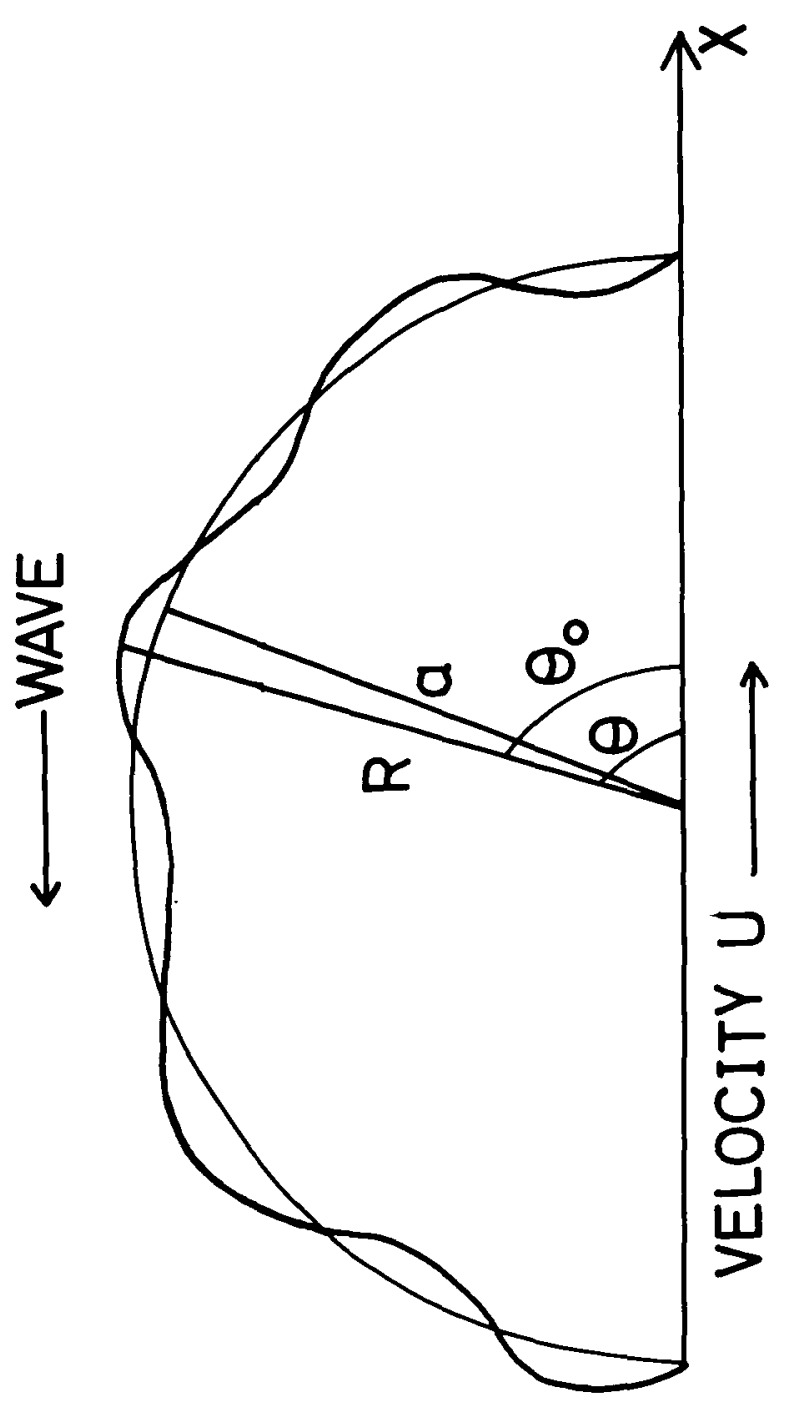

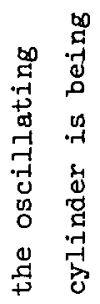

号壱

gृ

की

$\stackrel{0}{7}$

○े 今े

का क

$\rightarrow$ c.

\& न

舢

.न

ro 0.

ป

马 离

吾

$\stackrel{\pi}{+} \stackrel{5}{\circ}$

का

:

¿

की 9

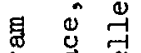

कृ

落

$-\dot{ }$

崩 
the introduction that it is more convenient to use a moving origin, so we add $\psi=-U r \sin \theta$ to the solution for the stream function equation. Thus the solution for the stream function which allows this finite velocity of propulsion $U$ is,

$$
\psi=-U r \sin \theta+a_{0} \theta+\frac{a_{1}}{r} \sin \theta+\sum_{n=2}^{\infty}\left(\frac{a_{n}}{r^{n}}+\frac{b_{n}}{r^{n-2}}\right) \sin \theta .
$$

On satisfying the boundary conditions on the cylinder of radius $a$, we obtain the following solutions for the velocities in terms of the surface coefficients.

$$
\text { (7) } \begin{aligned}
u=-U \cos \theta+\frac{a A_{0}}{r}+\frac{1}{2}\left(A_{1}+B_{1}\right) \frac{a^{2}}{r^{2}} \cos \theta+\sum_{n=2}^{\infty} & \frac{1}{2} A_{n} \cos n \theta\left[n \frac{a^{n-1}}{n-1}-(n-2) \frac{a^{n+1}}{r^{n+1}}\right] \\
& +\sum_{n=2}^{\infty} \frac{n}{2} B_{n} \cos n \theta\left[\frac{a^{n+1}}{r^{n+1}}-\frac{a^{n-1}}{r^{n-1}}\right]
\end{aligned}
$$

and

(8) $v=U \sin \theta+\frac{1}{2}\left(A_{1}+B_{1}\right) \frac{a^{2}}{r^{2}} \sin \theta+\sum_{n=2}^{\infty} \frac{1}{2}(n-2) A_{n} \sin n \theta\left[\frac{a^{n-1}}{r^{n-1}}-\frac{a^{n+1}}{r^{n+1}}\right]$

$$
+\sum_{n=2}^{\infty} \frac{1}{2} B_{n} \sin n \theta\left[\frac{a^{n+1}}{r^{n+1}}-(n-2) \frac{a^{n-1}}{r^{n-1}}\right] \text {. }
$$

The velocity of propulsion is defined in terms of the first two coefficients $A_{1}$ and $B_{1}$ in (4) by,

$$
U=\frac{1}{2}\left(B_{1}-A_{1}\right) \text {. }
$$

This may be compared to a similar result obtained for a sphere by Lighthill [3] and Blake [1].

Other physical quantities which have applications to this problem are the pressure and the stresses exerted by the body on the fluid; these being found in terms of the surface velocity coefficients. The solution for the pressure is,

$$
p=2 \mu \sum_{n=2}^{\infty}(n-1) \frac{a^{n-1}}{r^{n}}\left(A_{n}-B_{n}\right) \cos n \theta,
$$

which is found by substitution of the velocity component solutions $((7)$ and 
(8)) into the creeping flow equation (1). The surface stresses $\sigma_{i j}$ exerted by the body on the fluid are defined by,

$$
\sigma_{i j}=p \delta_{i j}-2 \mu e_{i j},
$$

where $e_{i j}$ is the rate of strain tensor. After substitution, equation (11) yields the following stresses on the fluid,

$$
\begin{aligned}
\sigma_{r P}=2 \mu\left[\left(A_{1}+B_{1}\right) \frac{a^{2}}{r^{3}}\right. & \cos \theta+\frac{a A_{0}}{r^{2}} \\
& +\sum_{n=2}^{\infty} \frac{1}{2} A_{n} \cos n \theta\left[(n+2)(n-1) \frac{a^{n-1}}{r^{n}}-(n-2)(n+1) \frac{a^{n+1}}{r^{n+2}}\right] \\
& \left.+\sum_{n=2}^{\infty} \frac{1}{2} B_{n} \cos n \theta\left[n(n+1) \frac{a^{n+1}}{r^{n+2}}-(n-1)(n+2) \frac{a^{n-1}}{r^{n}}\right]\right],
\end{aligned}
$$

$$
\begin{aligned}
\sigma_{\theta \theta}=-2 \mu\left[\left(A_{1}+B_{1}\right) \frac{a^{2}}{r^{3}}\right. & \cos \theta+\frac{a A_{0}}{r^{2}} \\
& +\sum_{n=2}^{\infty} \frac{1}{2}(n-2) A_{n} \cos n \theta\left[(n-1) \frac{a^{n-1}}{r^{n}}-(n+1) \frac{a^{n+1}}{r^{n+2}}\right] \\
& \left.+\sum_{n=2}^{\infty} \frac{1}{2} B_{n} \cos n \theta\left[n(n+1) \frac{a^{n+1}}{r^{n+2}}-(n-1)(n-2) \frac{a^{n-1}}{r^{n}}\right]\right],
\end{aligned}
$$

(14) $\sigma_{r \theta}=\mu\left[2\left(A_{1}+B_{1}\right) \frac{a^{2}}{r^{3}} \sin \theta\right.$

$$
\begin{aligned}
& +\sum_{n=2}^{\infty} A_{n} \sin n \theta\left[n(n-1) \frac{a^{n-1}}{r^{n}}-(n-2)(n+1) \frac{a^{n+1}}{r^{n+2}}\right] \\
& \left.+\sum_{n=2}^{\infty} n B_{n} \sin n \theta\left[(n+1) \frac{a^{n+1}}{r^{n+2}}-(n-1) \frac{a^{n-1}}{r^{n}}\right]\right] .
\end{aligned}
$$

These equations then enable us to calculate the rate of working per unit area $P$, which is defined by the following integral,

$$
P=\frac{1}{2 \pi} \int_{0}^{2 \pi}\left(u \sigma_{r r}+v \sigma_{r \theta}\right)_{r=a} d \theta .
$$

This on evaluation yields, 


$$
P=\frac{\mu}{a} \sum_{n=1}^{\infty}\left[n\left(A_{n}^{2}+B_{n}^{2}\right)+2 A_{n}^{B} n\right] .
$$

We define an efficiency for this model by the formula,

$$
\eta=\frac{\overline{T U}}{\bar{P}},
$$

where $\eta$ is the efficiency $(\%), \bar{T}$ an average characteristic thrusting force per unit area, $\bar{U}$ the velocity of propulsion and $\bar{P}$ the mean rate of working defined in (15).

An average characteristic thrusting force per unit area $(\bar{T})$ can be obtained by taking a suitable average of the stresses over the oscillating surface of the organism. It is found that this thrusting force is proportional to $\mu U / a$. There is, of course, an equal and opposite resistance force which balances this thrusting force. However, we will defer calculation of this force until the oscillatory surface is defined because we need to take $T$ over this surface.

\section{Surface conditions and velocity of propulsion}

The oscillatory surface of the cylinder is defined by,

$$
R=a\left[1+\varepsilon \sum_{n=2}^{N} \alpha_{n}(t) \cos n \theta\right]
$$

$$
\theta_{0}=\theta+\varepsilon \sum_{n=1}^{N} \beta_{n}(t) \sin n \theta
$$

where $\alpha_{n}(t)$ and $\beta_{n}(t)$ are periodic functions in time and $\varepsilon$ is suitably small for a small parameter expansion. To obtain the surface velocity coefficients $A_{n}$ and $B_{n}$, an iterative technique is employed by using a Taylor series expansion for the velocity components.

(19) $u(a, \theta)=u^{[N]}(a, \theta)$

$$
=u\left(R, \theta_{0}\right)-\sum_{\substack{m=1 \\
m=p+q}}^{N-1}\left(\begin{array}{l}
m \\
p
\end{array}\right) \frac{(R-a)^{p}\left(\theta_{0}-\theta\right)^{q}}{m !}\left[\frac{\partial^{m} u^{[N-m]}}{\partial r^{p} \partial \theta^{q}}\right]_{r=a}+O\left(\varepsilon^{N+1}\right),
$$


where $u^{[N]}(a, \theta)$ is the $N$-th approximation to the velocity on the cylinder $(a, \theta) \cdot \mathbf{u}\left(R, \theta_{0}\right)$ is the velocity vector at the oscillating surface and is easily obtained by taking the time derivative of (18). The series term on the right hand side of (18) is the Taylor series expansion about $(a, \theta)$.

The first approximation can be obtained from the velocities at the oscillating surface due to the no-slip condition.

$$
u\left(R, \theta_{0}\right)=\dot{R}=a \varepsilon \sum_{n=2}^{N} \dot{\alpha}_{n} \cos n \theta
$$

and

$$
v\left(R, \theta_{0}\right)=R \dot{\theta}_{0}=\alpha \varepsilon\left(1+\varepsilon \sum_{n=2}^{N} \alpha_{n} \cos n \theta\right) \sum_{n=1}^{N} \dot{\beta}_{n} \sin n \theta
$$

yields the following components for the first approximation:

$$
A_{n}=a \varepsilon \dot{\alpha}_{n}, \quad B_{n}=a \varepsilon \dot{\beta}_{n}, \quad(n=1,2, \ldots, N) \text {. }
$$

Because $B_{1}$, to the first approximation, is purely periodic in time, the velocity of propulsion is zero $\left(A_{1}=0\right.$, to first approximation, as $\alpha_{1}=0$ ).

To obtain the second approximation for both $A_{n}$ and $B_{n}$, we substitute into the Taylor series expansion (19) as follows,

(22) $u(a, \theta)=a \varepsilon \sum_{n=2}^{N} \dot{\alpha}_{n} \cos n \theta$

$$
\begin{array}{r}
+a \varepsilon^{2} \sum_{n=2}^{N} \alpha_{n} \cos n \theta\left[\dot{\beta}_{1} \cos \theta+\sum_{n=2}^{N}\left(\dot{\alpha}_{n}+n \dot{\beta}_{n}\right) \cos n \theta\right] \\
+a \varepsilon^{2} \sum_{n=1}^{N} \beta_{n} \sin n \theta\left[\sum_{n=2}^{N} n \dot{\alpha}_{n} \sin n \theta\right]
\end{array}
$$

and 
(23) $v(a, \theta)=a \varepsilon\left(1+\varepsilon \sum_{n=2}^{N} \alpha_{n} \cos n \theta\right) \sum_{n=1}^{N} \dot{\beta}_{n} \sin n \theta$

$$
\begin{array}{r}
+a \varepsilon^{2} \sum_{n=2}^{N} \alpha_{n} \cos n \theta\left[\dot{\beta}_{1} \sin \theta-\sum_{n=2}^{N}\left\{(n-2) \dot{\alpha}_{n}-(2 n-1) \dot{\beta}_{n}\right\} \sin n \theta\right] \\
-a \varepsilon^{2} \sum_{n=1}^{N} \beta_{n} \sin n \theta \sum_{n=1}^{N} n \dot{\beta}_{n} \cos n \theta .
\end{array}
$$

From these equations we can calculate $A_{n}$ and $B_{n}$ to second order. The important values are $A_{1}$ and $B_{1}$ which enable us to derive the second order velocity of propulsion

$$
\begin{aligned}
& U=\frac{1}{2} a \varepsilon \dot{\beta}_{1}+\frac{1}{4} a \varepsilon^{2}\left[2 \beta_{1} \dot{\beta}_{2}-\beta_{2} \dot{\beta}_{1}-3 \alpha_{2} \dot{\beta}_{1}-2 \beta_{1} \dot{\alpha}_{2}\right. \\
& +\sum_{n=2}^{N-1}\left\{(n+1) \alpha_{n} \dot{\beta}_{n+1}-n \dot{\alpha}_{n} \beta_{n+1}-3 n \alpha_{n+1} \dot{\beta}_{n}-(n+1) \dot{\alpha}_{n+1} \beta_{n}+(n-3) \dot{\alpha}_{n} \alpha_{n+1}-n \alpha_{n} \dot{\alpha}_{n+1}\right. \\
& \left.\left.+(n+1) \beta_{n} \dot{\beta}_{n+1}-n \beta_{n+1} \dot{\beta}_{n}\right\}\right] .
\end{aligned}
$$

As $\alpha_{n}$ and $\beta_{n}$ are purely periodic in time, we need to go to this second order approximation to obtain a mean velocity of propulsion $\vec{U}$.

\section{Examples of models}

In the five simple examples considered in this section, two can be thought of in terms of a standing wave form, whereas the other three have a progressive wave character. These models are best defined by the following equation for the surface shape,

$$
\begin{aligned}
R & =a\left[1+\varepsilon_{1} \cos \sigma t \cos N \theta+\varepsilon_{2} \sin \sigma t \cos (N+1) \theta\right] \\
\theta_{0} & =\theta+\varepsilon_{3} \cos \sigma t \sin N \theta+\varepsilon_{4} \sin \sigma t \sin (N+1) \theta .
\end{aligned}
$$

For these particular models, it is found that the average characteristic thrusting force per unit area $T$ which is extracted from the stress integral is equal to $2 \mu(N / a) U$. This can be compared to the thrusting force employed in Blake [2] for other infinite models of micro-organism propulsion where the thrusting force was equal to $2 \mu k U$ ( $k$ is the wave number). For the above models the equivalent wave number is $N / a$, so it can be seen that this compares with the results we would expect from previous experience. Thus the efficiency is defined for the models 
discussed above by

$$
\eta=\frac{2 \mu N / a \bar{U}^{2}}{\bar{P}}
$$

The following table gives the values of $\varepsilon_{i}(i=1,2,3,4)$, velocity of propulsion and efficiency for $N=20$ and $\varepsilon=.025$.

\begin{tabular}{crrrrrr} 
Models & $\varepsilon_{1} / \varepsilon$ & $\varepsilon_{2} / \varepsilon$ & $\varepsilon_{3} / \varepsilon$ & $\varepsilon_{4} / \varepsilon$ & $U / \frac{1}{8} \alpha \varepsilon^{2} \sigma$ & $n(\%)$ \\
\hline$(1)$ & 1 & 0 & 0 & 1 & $2 N+1$ & 3.2 \\
$(2)$ & -1 & 1 & 0 & 0 & $2 N-3$ & 2.6 \\
$(3)$ & 0 & 0 & -1 & 1 & $-(2 N+1)$ & 3.2 \\
$(4)$ & 0 & 1 & 1 & 0 & $2 N-1$ & 2.9 \\
$(5)$ & -.707 & .707 & -.707 & .707 & $-(2 N+2)$ & 3.2 \\
\hline
\end{tabular}

Figure 2. Table of velocity of propulsion and efficiency for models defined by (25)

It should be emphasised that these values of the velocity of propulsion are only valid for small $\varepsilon$, a better approximation being obtained if we go to fourth order. In models (2), (3) and (5) the wave is travelling to the left (that is, increasing $\theta$ for $0 \leq \theta \leq \pi$ ). It is noticed that models (2) and (3) propel themselves in opposite direction relative to the wave, which is anticipated from Blake [1] and [2].

In conclusion, a solution to flow around a circular cylinder at low Reynolds number is obtained which has interesting comparisons to the classic stokes paradox problem. It has been shown that a cylinder with wave motions on its surface can propel itself through a very viscous fluid.

\section{References}

[1] J.R. Blake, "A spherical envelope approach to ciliary propulsion", $J$. Fluid Mech. 46 (1971), 199-208.

[2] J.R. Blake, "Infinite models for ciliary propulsion", J. Fluid Mech. (to appear). 
[3] M.J. Lighthill, "On the squirming motion of nearly spherical deformable bodies through liquids at very small Reynolds numbers", Comm. Pure App 2. Maths. 5 (1952), 109-118.

Department of Applied Mathematics and Theoretical Physics, University of Cambridge,

Cambridge, England. 